\title{
Preliminary evidence of immune function modulation by thyroid hormones in healthy men and women aged 55-70 years
}

\author{
Clare F Hodkinson', Ellen E A Simpson², John H Beattie ${ }^{3}$, Jacqueline M O'Connor ${ }^{1}$, \\ David J Campbell ${ }^{1}$, J Strain ${ }^{1}$ and Julie M W Wallace ${ }^{1}$ \\ ${ }^{1}$ Northern Ireland Centre for Food and Health (NICHE) and ${ }^{2}$ School of Psychology, University of Ulster, Coleraine, Northern Ireland BT52 1SA, UK \\ ${ }^{3}$ Rowett Research Institute, Bucksburn, Aberdeen, Scotland AB21 9SB, UK \\ (Correspondence should be addressed to J M W Wallace; Email: j.wallace@ulster.ac.uk)
}

\begin{abstract}
A reciprocal relationship between the endocrine and immune system has been demonstrated under pathophysiological conditions. However, few studies have assessed the relationship between thyroid hormones and immune function in apparently healthy individuals. Therefore, to clarify our understanding of normal physiological endocrine-immune interactions this study aimed to examine the interrelationships between thyroid hormones and immunity in healthy individuals. Total triiodothyronine $\left(\mathrm{T}_{3}\right)$, total thyroxine $\left(\mathrm{T}_{4}\right)$ and markers of immune status were assessed in 93 free-living and apparently healthy individuals aged $55-70$ years. $\mathrm{T}_{3}$ and $\mathrm{T}_{4}$ concentrations were determined by commercially available kits. Immune status was assessed using flow cytometry and biochemical markers. Statistical analysis was performed by partial correlation, controlling for age. Thyroid hormone concentration was positively associated with markers of inflammation $(P \leq 0 \cdot 05)$, natural killer-like $\mathrm{T}$ cells
\end{abstract}

$(P \leq 0 \cdot 001)$, expression of interleukin-6 (IL6) by activated monocytes $(P \leq 0 \cdot 05)$; percentage expression of memory T-lymphocytes $(P \leq 0 \cdot 01)$, memory T-helper lymphocytes $(P \leq 0 \cdot 05)$ and memory T-cytotoxic lymphocytes $(P \leq 0 \cdot 05)$, and higher IL2 receptor density on CD3 + T-lymphocytes $(P \leq 0 \cdot 05)$. Thyroid hormone concentration was inversely associated with early lymphocyte apoptosis $(P \leq 0 \cdot 05)$ and the ratio of naïve- to memory T-cytotoxic lymphocytes $(P \leq 0 \cdot 05)$. The current study provides preliminary evidence of a role for $\mathrm{T}_{3}$ and $\mathrm{T}_{4}$, within normal physiological ranges, in the maintenance of lymphocyte subpopulations, and in mediating the inflammatory response. In conclusion, these findings highlight the potential implications of altered thyroid function in older individuals and the importance of future research examining thyroid-immune interactions.

Journal of Endocrinology (2009) 202, 55-63

\section{Introduction}

Bidirectional communication between the central nervous system and immune system is mediated by the endocrine system, via the hypothalamic-pituitary-adrenal (HPA) axis (Felten \& Felten 1991, Bellinger et al. 2002). Evidence, predominantly from animal studies, also suggests a direct reciprocal relationship exists between the thyroid gland and the immune system (Fabris et al. 1995, Klein 2006). Thyroid hormones are involved in numerous physiological processes, such as development, growth and the metabolism of lipids and carbohydrates. While it is believed thyroid hormones are not critical to the development of a normal immune response, they are thought to be involved in the maintenance of immune function in response to environmental stimuli and stress-mediated immunosuppression (Davis 1998, Dorshkind \& Horseman 2000, Dorshkind \& Horsemanelson 2001). Total triiodothyronine $\left(\mathrm{T}_{3}\right)$ is able to modulate immune function via nuclear receptors (TRs) to regulate target genes, and by non-genomic interactions with membrane receptors independent of protein synthesis (Yen 2001, Csaba et al. 2005, Pallinger et al. 2005); the mechanisms that are speculated to underlie the aspects of immunity such as the homing of thymocytes to peripheral lymphoid organs (RibeiroCarvalho et al. 2007), and immune response to vaccination (Klecha et al. 2000). The prevalence of thyroid function disorders increases with age (Mariotti et al. 1995, Chiovato et al. 1997, Latrofa \& Pinchera 2005), and hypothalamicpituitary-thyroid (HPT) dysfunction and altered thyroid metabolism occurs even in apparently healthy older individuals (Mooradian 1995, Chakraborti et al. 1999, Magri et al. 2002). Given the relationship between thyroid and immune function, alterations in thyroid hormone concentration with age may contribute to age-related changes in immune function (Stulnig et al. 1995, McNerlan et al. 1999, Santagostino et al. 1999, Bisset et al. 2004).

Previous investigation of the relationship between thyroid hormones and immune functions has been predominantly restricted to a limited range of immune markers in clinical populations with thyroid function disorders (Palmblad et al. 
1981, Nakanishi et al. 1991, Covas et al. 1992, Mariotti et al. 1992, Kretowski et al. 1999, Bossowski et al. 2003). While these studies demonstrate that altered thyroid hormone concentration is associated with altered immunity, it is less clear whether these observations are directly due to the actions thyroid hormones or underlying autoimmunity. As age is a significant factor in the incidence of thyroid disorders, the current study aimed to determine if thyroid hormone concentrations, under normal physiological conditions in healthy older individuals, mirrors the thyroidimmune alterations observed in clinical populations. The use of a comprehensive range of phenotypic and functional immune markers was employed to provide a more complete assessment of potential immune modulation by thyroid hormones.

\section{Material and Methods}

\section{Participants}

Individuals aged 55-70 years were recruited through media coverage, and leaflets, as well as national and local organizations with members spanning this age group. Individuals were screened based on defined exclusion criteria, adapted from the SENIOR protocol (Ligthart et al. 1984) which included: body mass index (BMI; <20 and $>33 \mathrm{~kg} / \mathrm{m}^{2}$ ); abnormal haematology, liver and kidney function tests; dietary habits (for e.g. vegetarians and vegans); depression (score $\geq 11$ on the geriatric depression scale); dementia (score $\leq 24$ on the Mini Mental State Examination); pre-menopausal women; no more than three drugs per day including the use of antidepressants, laxatives, hormonal replacement therapy or other immune modulating medication; diagnosis of or treatment for pathological diseases (cancer, diabetes, insufficient renal and hepatic performance, metabolic, malabsorption and chronic inflammatory pathologies); habitual use of vitamin and/or mineral supplements within the last 6 months; alcohol consumption $(>30 \mathrm{~g} /$ day for men and $>20 \mathrm{~g} /$ day for women); and smoking $(>10$ cigarettes, cigars or pipe/day). Of the individuals screened, $15 \%$ (147) were invited to participate. Ninety-three apparently healthy late-middle aged individuals (48 females and 45 males) aged 55-70 years completed the study. The University of Ulster Research Ethical Committee granted approval for the study. All volunteers gave written informed consent in accordance with the declaration of Helsinki.

\section{Experimental protocol and sample collection}

Following an overnight ( $>12 \mathrm{~h}$ ) fast, participants were asked to attend the research centre at $0830 \mathrm{~h}$ on the study day. Anthropometric measurements were undertaken and blood was collected. Height and weight was measured and used to calculate BMI as body weight $(\mathrm{kg})$ divided by height $(\mathrm{m})$ squared. Fasting, venous blood $(55 \mathrm{ml})$ was collected into vacutainers containing $\mathrm{K}_{3}$ EDTA and sodium-heparin and into serum separator tubes. $\mathrm{K}_{3}$ EDTA and sodium-heparin whole blood was used for determination of immune status on a FACSCalibur flow cytometer (BD Biosciences, Oxford, UK) within $4 \mathrm{~h}$ of collection. $\mathrm{K}_{3}$ EDTA anti-coagulated whole blood was also used to assess full blood profiles, which were conducted at the Causeway Laboratory, Causeway NHSS Trust, Coleraine, UK. Serum was separated and stored at $-80{ }^{\circ} \mathrm{C}$ until analysis at the end of the study.

Table 1 Anti-human conjugated monoclonal antibodies used in FACS analysis of immune markers in healthy individuals, aged 55-70 years

Marker

Antibody
Simultest $\gamma_{1} / \gamma_{1}$
Intracellular $\gamma_{1}$
Intracellular $\gamma_{2}$
CD3
CD14
CD19
Simultest CD3/CD16/CD56
CD25
CD3/anti-HLA-DR
CD4
CD8
CD45RO
CD45RA
IL-1 $\beta$
IL6
Annexin V
PI
PHACOTEST $E$. coli

Negative control
Negative control
Negative control
Pan T lymphocyte
Monocyte
B lymphocyte
NK and NKT cells
Intermediately activated cells (IL-2R)
Late-activated T lymphocytes
T-helper lymphocytes
T-cytotoxic lymphocytes
Memory cells
Naïve cells
Interleukin-1 $\beta$
Interleukin-6
Early cellular apoptosis
Late cellular apoptosis/necrosis
Phagocytic capacity/activity

Clone

X40, X40
X40
X39
SK7
M\$P9
SK7, SJ25C1
SK7, B73·1, MY31
2A3
SK7, L243
SK3
SK1
UCHL-1
L48
AS10
AS12
-
-
-

\begin{tabular}{l} 
Conjugate \\
\hline \\
FITC/PE \\
PE \\
FITC \\
FITC and PE \\
FITC \\
FITC and PE \\
FITC/PE/PE \\
PE \\
FITC/PE \\
PerCP \\
PerCP \\
PE \\
FITC \\
PE \\
PE \\
FITC \\
- \\
FITC
\end{tabular}

FACS, fluorescence activated cell sorter; CD, cluster of differentiation; FITC, fluorescein; PE, phycoerythrin; NK, natural killer; HLA, human leukocyte antigen; $\mathrm{DR}$, D- related; PerCP, peridinin chlorphyll protein; IL, interleukin; PI, propidium iodide. All products were supplied by BD BioSciences, Oxford, UK, with the exception of PHAGOTEST Escherichia coli, which was supplied by ORPEGEN Pharma, Heidelberg, Germany. 

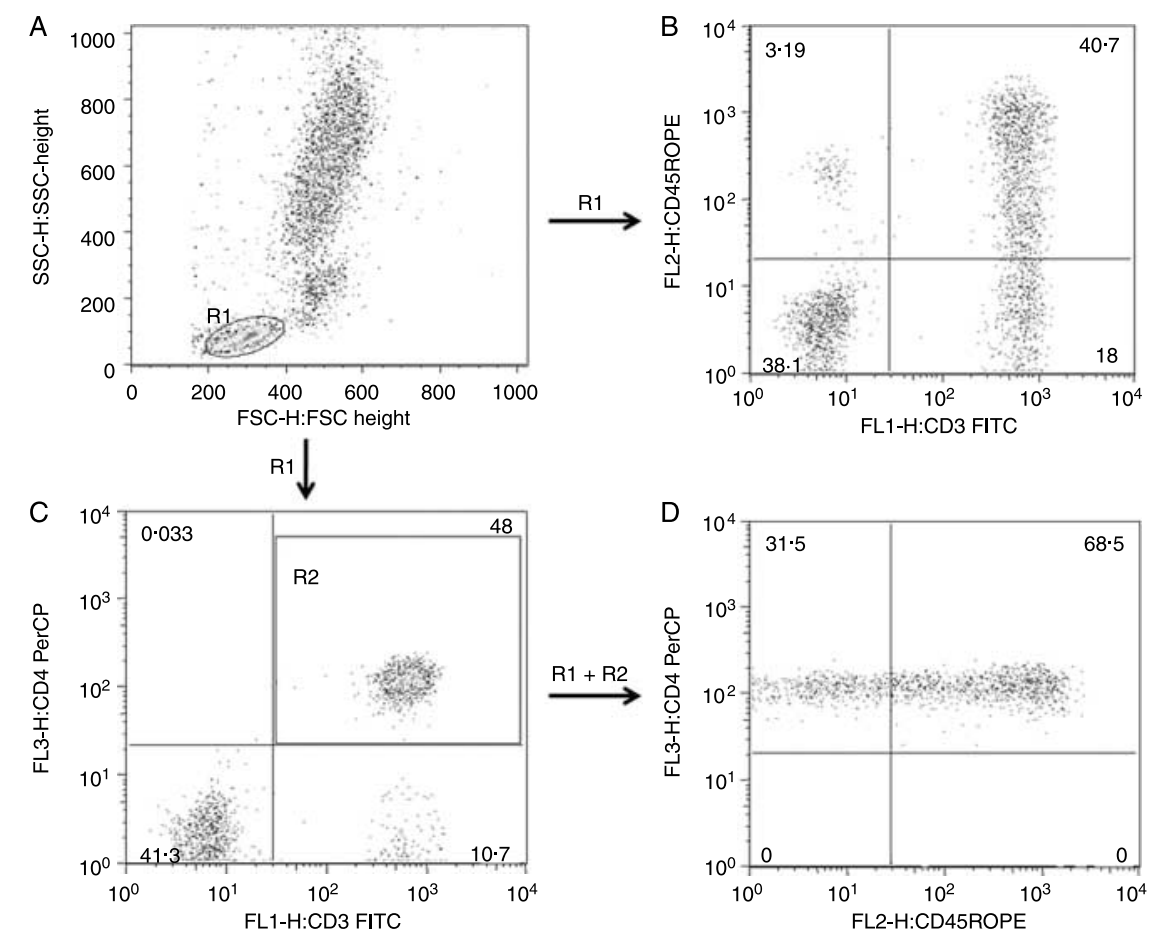

Figure 1 Gating strategy for the three-colour analysis of lymphocyte subsets. (A) Lymphocytes (R1) were identified by FSC versus SSC scatter characteristics excluding debris, monocytes and granulocytes. (B) Gating on R1 facilitated the determination of CD3+/CD45RA+ or $\mathrm{CD} 3+/ \mathrm{CD} 45 \mathrm{RO}+$ lymphocytes subsets. (C) CD3 +/CD4 + (or CD3 +/CD8+) lymphocytes were identified by gating on $\mathrm{R} 1$; a second gate (R2) was placed around CD3+CD4+ lymphocytes. (D) Gates R1 + R2 enabled the determination of CD3 +/CD4 +/CD45RO+ or CD45RA + lymphocytes. The same gating strategy was applied for the determination of CD3 +/CD8 + lymphocyte subsets.

\section{Thyroid hormones}

Serum $T_{3}$ and total thyroxine $\left(T_{4}\right)$ were measured using a competitive immunoassay with an enhanced chemiluminescence end point (Vitros Immunodiagnostic Products, Ortho-clinical Diagnostics, Amersham, UK).

\section{Inflammatory markers}

Serum was analysed for C-reactive protein (CRP-high sensitivity), complement protein-3 (C3), and complement protein-4 (C4), using commercially available kits on a Hitachi 912 analyzer (Roche Diagnostics). Serum ferritin was determined at Grenoble University Hospital by sensitized immunoprecipitation using automatic nephelometry (BNII nephelometer; Dade Behring, Paris La De'fence, France) as per the manufactures guidelines; laboratory quality assurance included analysis of serum from standard pools and international standards with each run.

\section{Leukocyte immunophenotyping}

Immunophenotyping of leukocyte subsets was carried out by two- and three-colour flow cytometry using panels of conjugated-monoclonal antibodies (mAb; Table 1). $\mathrm{K}_{3}$ EDTA whole blood $(100 \mu \mathrm{l})$ was incubated with conjugated-mAbs using a lyse/wash protocol (BD Biosciences). Samples were analysed within $4 \mathrm{~h}$ of fixation. Two-colour analysis was performed for the following lymphocyte subsets: CD3 + T-lymphocytes; CD3 - /CD19+B-lymphocytes; CD3-/ $\mathrm{CD}(16+56)+$ natural killer (NK) cells; $\mathrm{CD} 3+/ \mathrm{CD}(16$ +56) + NK-like T (NKT) cells; CD3 + /CD25 + intermediately-activated T-lymphocytes; and CD3+/human leukocyte antigen-DR + late-activated T-lymphocytes with the gating of lymphocytes (R1) using scatter characteristics (forwardscatter (FSC) versus side-scatter (SSC)) to facilitate the exclusion of monocytes and granulocytes from analysis. Three-colour analysis of CD $3+/ \mathrm{CD} 4+$ total T-helper lymphocytes, $\mathrm{CD} 3+/ \mathrm{CD} 8+$ total cytotoxic- $\mathrm{T}$ lymphocytes (CTL), CD3 + /CD4 + /CD45RA + naïve T-helper lymphocytes, CD3 +/CD4+/CD45RO+memory T-helper lymphocytes, $\mathrm{CD} 3+/ \mathrm{CD} 8+/ \mathrm{CD} 45 \mathrm{RA}+$ naïve $\mathrm{CTL}$ and $\mathrm{CD} 3+/ \mathrm{CD} 8+/ \mathrm{CD} 45 \mathrm{RO}+$ memory $\mathrm{CTL}$ was performed by gating lymphocytes (R1) using scatter characteristics (FSC versus SSC) to facilitate the exclusion of monocytes and granulocytes from analysis, and either CD3 + CD4 + events (R2) or CD3 + CD8 + events to determine further CD45RO 
or CD45RA T-lymphocyte subsets, see Fig. 1a-d. The percentage of positive cells was reported as obtained from fluorescence activated cell sorter (FACS) analysis and absolute counts were calculated from the lymphocyte white blood cell differential $\left(\times 10^{9} / 1\right)$. Ratios of lymphocyte subsets were calculated from absolute counts.

\section{Apoptosis}

The determination of early lymphocyte apoptosis by twocolour flow cytometry was conducted using the Annexin V-fluorescein (FITC) Apoptosis Detection Kit I (BD Biosciences). Briefly, peripheral blood mononuclear cells (PBMC) were separated by density gradient centrifugation from sodium-heparin anti-coagulated whole blood using Accuspin System-Histopaque-1077 tubes (Sigma-Aldrich). PBMC were washed twice in fresh sterile filtered PBS and then re-suspended in buffer. PBMC were stained using Annexin V-FITC and propidium iodide (PI). Lymphocytes were gated using a FSC versus SSC dotplot. Lymphocytes staining positive for Annexin-V and negative for PI were determined using fluorescence channel 1-height (FL1-H) versus fluorescence channel 2-height (FL2-H) dotplots (see Fig. $2 \mathrm{a}-\mathrm{c}$ ). The percentage of lymphocytes undergoing early apoptosis was obtained from FACS analysis, and absolute counts were calculated using the lymphocyte white blood cell differential $\left(\times 10^{9} / 1\right)$.

\section{Phagocytosis}

The quantification of phagocytic capacity and activity of granulocytes and monocytes was determined using a PHAGOTEST kit (ORPEGEN Pharma, Heidelberg, Germany). Sodium-heparin anti-coagulated whole blood $(100 \mu \mathrm{l})$ was cooled on ice for $10 \mathrm{~min}$, prior to incubation with opsonized Escherichia coli-FITC in capped Falcon tubes (BD Biosciences) for $10 \mathrm{~min}$ at $37^{\circ} \mathrm{C}$ with $5-7 \% \mathrm{CO}_{2}$. Monocytes and granulocytes were gated using a FSC versus SSC dotplot. SSC versus E. coli-FITC dotplots were used to measure phagocytic capacity and activity for both cell types (see Fig. 3a-e). Percentage of E. coli-FITC-positive cells was used to determine the capacity of monocytes and granulocytes to conduct phagocytosis. Mean fluorescence intensity (MFI) was used as a quantitative measure to determine the number of $E$. coli ingested per cell; this measure was used as a determinant of phagocytic activity.

\section{Determination of intracellular cytokine production by activated monocytes}

To determine intracellular cytokine production by activated monocytes, $1 \mathrm{ml}$ sodium-heparin anti-coagulated whole blood was activated by incubation with lipopolysaccharide at $1 \mu \mathrm{g} / \mathrm{ml}$ and secretion of intracellular cytokines blocked with brefeldin A at $10 \mu \mathrm{g} / \mathrm{ml}$ (Sigma-Aldrich) for $4 \mathrm{~h}$ at $37^{\circ} \mathrm{C}$ with $5-7 \% \mathrm{CO}_{2}$. After activation, $100 \mu \mathrm{l}$ activated blood was incubated with either $10 \mu \mathrm{lgG} 2 \alpha$ FITC (isotype control) or CD14 FITC (BD Pharmingen, Oxford, UK) for $30 \mathrm{~min}$ in the dark at room temperature. Erythrocytes were lysed by incubation with $100 \mu$ fixation medium A (Caltag, Invitrogen) for $30 \mathrm{~min}$ in the dark at room temperature. Cells were washed using cell wash solution (11 sterile PBS containing $0.5 \% \mathrm{BSA}$ and $0 \cdot 1 \% \mathrm{NaN}_{3}$ ), and were centrifuged at $300 \mathrm{~g}$ for $5 \mathrm{~min}$ at $4{ }^{\circ} \mathrm{C}$. Supernatant was removed, and the cell pellet re-suspended and incubated with $100 \mu \mathrm{l}$ permeabilizing medium B (Caltag, Invitrogen) for $15 \mathrm{~min}$ in the dark at room temperature. Cells were then incubated with either $(10 \mu \mathrm{l})$ of the isotype control IgG1-phycoerythrinphycoerythrin (PE), interleukin (IL1) $\beta$ PE, or IL6 $\mathrm{PE}$ for $30 \mathrm{~min}$ in the dark at room temperature. Cells were washed as previously described, and fixed with $500 \mu \mathrm{l}$ $1 \times$ Cell Fix solution (BD Biosciences). Samples were analyzed immediately.

Monocytes were gated using FSC versus SSC dotplots, and percentages of cytokine-positive CD14+ cells were obtained from FL1-H versus FL2-H dotplots (see Fig. $4 a-c$ ). Quantification of intracellular cytokine production, as determined by the antibody binding capacity, was achieved using PE QuantiBrite beads (BD Biosciences) for standardization of PE MFI.
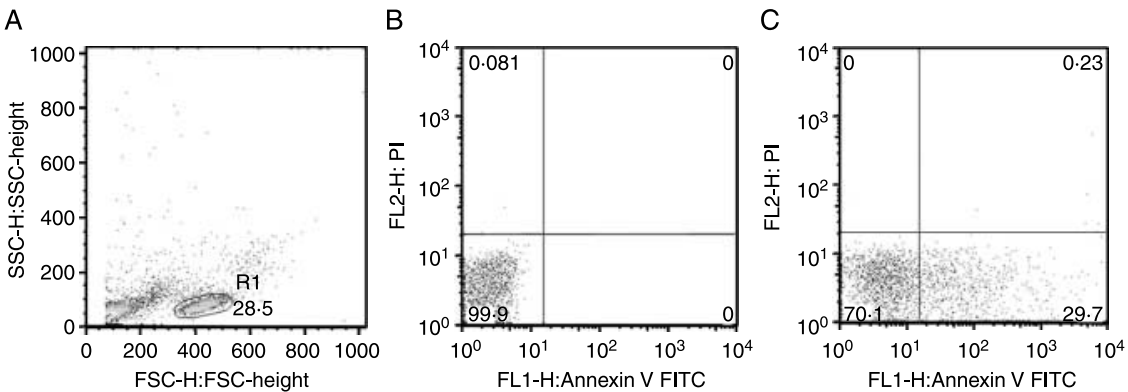

Figure 2 Gating strategy for two-colour analysis of early lymphocyte apoptosis. (A) Lymphocytes (R1) were identified by FSC versus SSC scatter characteristics. Dotplots B and C were gated on R1 and show the expression of Annexin $\mathrm{V}$ and $\mathrm{Pl}$ for the unstained negative control (B) and stained (C) test sample. 


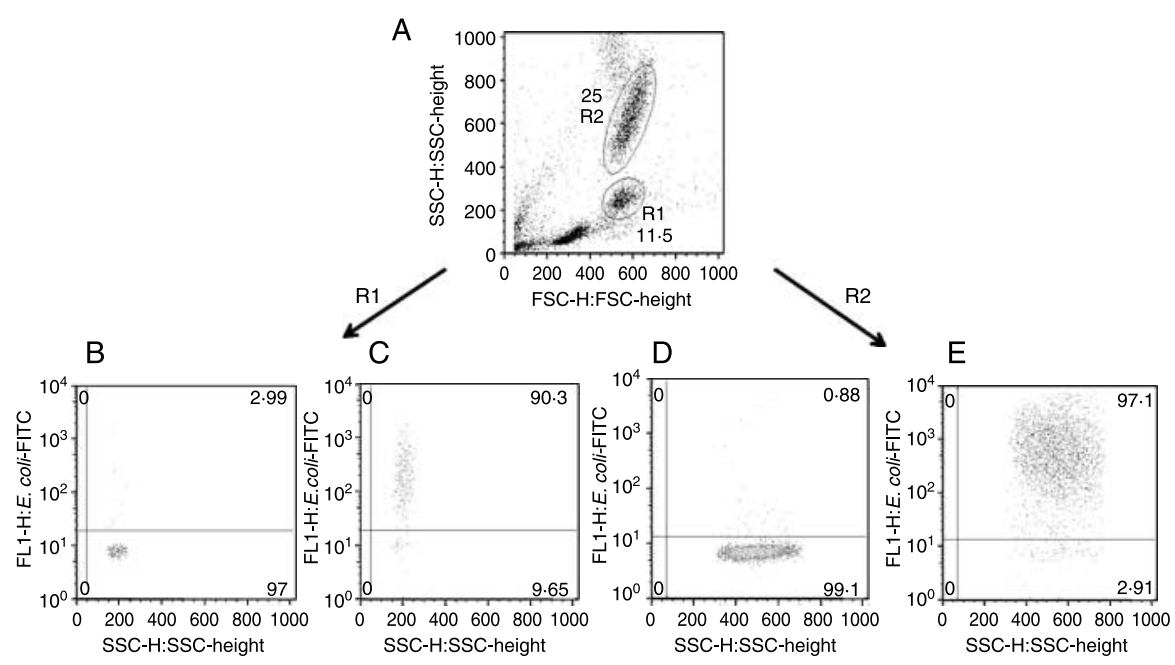

Figure 3 Gating strategy for one-colour analysis of phagocytic capacity and activity. The percentage of E. coli-FITC-positive cells was used to determine the capacity of monocytes or granulocytes to conduct phagocytosis. Mean fluorescence intensity (MFI) of E. coli-FITC-positive cells was used as a quantitative measure to determine the number of $E$. coli ingested per cell; this measure was used as a determinant of phagocytic activity. (A) FSC versus SSC scatter characteristics were used to identify monocyte (R1) and granulocyte (R2) populations. Expression of E. coli-FITC-positive R1-gated monocytes can be seen in control samples without $E$. coli-FITC incubation (B) and with incubation (C). Expression of E. coli-FITC-positive R2-gated granulocytes can be seen in control samples without E. coli-FITC incubation (D) and with incubation (E).

On each day of testing, three-colour calibration of the FACSCalibur was conducted using CaliBrite Beads-3 (BD Biosciences), and colour compensation levels using appropriate singly-stained cell suspensions. Flow cytometry data were acquired using CellQuest 3.3 for Power Macintosh (BD Biosciences) with 10000 gated events analysed per sample. Cytometric data was analysed using FlowJo (version 4.5) for Macintosh (TreeStar, Ashland, OR, USA).

\section{Statistical analysis}

Statistical analysis was performed using a Statistical Package for Social Sciences (SPSS; Chicago, IL, USA) version 11.0. Data were transformed to approximate normal distribution where appropriate. Sex-differences in thyroid hormone concentration were assessed by independent $t$-test $(P \leq 0 \cdot 05)$. As age was a significant determinant of immune function in this group (Hodkinson et al. 2006), the relationship between thyroid hormone concentration and immunity was explored using partial correlations, controlling for age.

\section{Results}

\section{Participants}

Participant characteristics are summarized in Table 2. At inclusion, volunteers were considered healthy and without incidence of infection in the preceding 4 weeks, as evidenced by self-reporting, a full blood profile, and normal liver and kidney function. Participants, were also unaffected by clinical depression and dementia. Participants had a mean age of $62 \cdot 4$ (S.D. $4 \cdot 48$ ) years and a mean BMI of $26 \cdot 9$ (s.D. $3 \cdot 22) \mathrm{kg} / \mathrm{m}^{2}$. There was no significant difference in age, BMI, $\mathrm{T}_{3}$ or $\mathrm{T}_{4}$ concentration between men and women.

\section{Thyroid hormones}

The euthyroid reference ranges for $\mathrm{T}_{3}$ and $\mathrm{T}_{4}$ were $1 \cdot 49-2 \cdot 60 \mathrm{nmol} / 1$ and $71 \cdot 2-141 \mathrm{nmol} / 1$ respectively. Mean ( \pm s.D.) values for the thyroid hormones $T_{3}, T_{4}$ and $T_{3}: T_{4}$ ratio were $1.95( \pm 0 \cdot 30) \mathrm{nmol} / \mathrm{l}, 85 \cdot 3( \pm 12 \cdot 5) \mathrm{nmol} / \mathrm{l}$ and $0 \cdot 23$ $( \pm 0 \cdot 003)$ ratio respectively, and were within normal range.

\section{Markers of inflammation and immunity}

Mean ( \pm s.D.) serum CRP, C3 and C4 for all subjects were $0 \cdot 20( \pm 0 \cdot 36) \mathrm{mg} / \mathrm{dl}, 1 \cdot 28( \pm 0 \cdot 21) \mathrm{g} / \mathrm{l}$ and $0 \cdot 26( \pm 0 \cdot 06) \mathrm{g} / \mathrm{l}$ respectively, and were within normal ranges. Ten participants had subclinical inflammation, as indicated by elevated serum CRP concentration $(>0.5 \mathrm{mg} / \mathrm{dl})$. There was no significant difference in ferritin or immune markers between participants with normal and elevated CRP concentration, with the exception of monocyte count, which were higher in individuals with raised CRP concentration $(P=0 \cdot 014)$. Medians (2.5-97.5 intervals) for all markers of immunity in this study population have previously been reported by Hodkinson et al. (2006). 

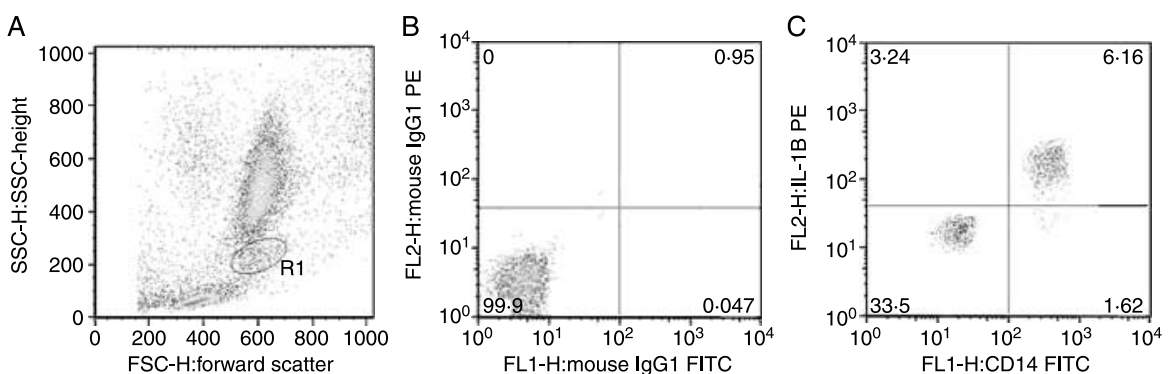

Figure 4 Gating strategy for two-colour analysis of lymphocyte intracellular cytokine expression and production by activated monocytes. Intracellular cytokine expression was determined from the percentage of IL-1 $\beta$ PE or IL6 PE positive monocytes. Quantification of intracellular cytokine production, as determined by the antibody binding capacity $(A B C)$, was achieved using PE QuantiBrite beads for standardization of PE MFI. (A) Monocytes (R1) were identified by FSC versus SSC scatter characteristics. Dotplots B and C were gated on R1 and show the expression of CD14 and IL-1 $\beta$ monocytes for the isotype negative control (B) and stained (C) test sample. The same gating strategy was applied for intracellular IL6 expression and production by activated monocytes.

\section{Associations between $T_{3}, T_{4}$ and markers of immunity}

Studies examining immune-endocrine interactions involving the hormones $T_{3}$ and $T_{4}$ have assessed a limited range of immune markers, predominantly in individuals with thyroid disorders which may be confounded by the presence underlying autoimmunity. Therefore, in the current study $\mathrm{T}_{3}$ and $\mathrm{T}_{4}$ were measured in healthy adults in addition to a comprehensive range of phenotypic and functional markers of immunity. As age was a significant determinant of immune function in this group it was necessary to include age as a co-founder in all analyses to control for any influence on immune function. Calculation of mean $T_{3}, T_{4}$ concentration, and $T_{3}: T_{4}$ ratio was used to discriminate between low- and high-thyroid hormone concentrations within normal physiological ranges.

Table 3 shows the significant associations between thyroid hormones and markers of immune function. $\mathrm{T}_{3}$ concentration was closely related to immune markers involved in innate immunity. Higher $T_{3}$ concentrations $(>1.95 \mathrm{nmol} / \mathrm{l})$ were

Table 2 Participant characteristics

\begin{tabular}{|c|c|c|}
\hline & Males $(n=45)$ & Females $(n=48)$ \\
\hline Age (years) & $62 \cdot 8 \pm 4 \cdot 68$ & $62 \cdot 2 \pm 2 \cdot 32$ \\
\hline $\mathrm{BMI}\left(\mathrm{kg} / \mathrm{m}^{2}\right)$ & $27 \cdot 2 \pm 2 \cdot 8$ & $26 \cdot 7 \pm 3 \cdot 55$ \\
\hline WBC $\left(\times 10^{9} / \mathrm{I}\right)$ & $5 \cdot 48 \pm 1 \cdot 28$ & $5 \cdot 10 \pm 1 \cdot 09$ \\
\hline $\mathrm{T}_{3}(\mathrm{nmol} / \mathrm{l})$ & $1 \cdot 89 \pm 0 \cdot 22$ & $1 \cdot 99 \pm 0.36$ \\
\hline $\mathrm{T}_{4}(\mathrm{nmol} / \mathrm{l})$ & $85 \cdot 6 \pm 11 \cdot 9$ & $85 \cdot 0 \pm 13 \cdot 1$ \\
\hline $\mathrm{T}_{3}: \mathrm{T}_{4}$ ratio & $0 \cdot 022 \pm 0 \cdot 003$ & $0 \cdot 024 \pm 0 \cdot 003$ \\
\hline $\begin{array}{l}\text { Afternoon }(1430 \mathrm{~h}) \\
\text { salivary cortisol } \\
(\mathrm{nmol} / \mathrm{l})^{\mathrm{a}}\end{array}$ & $5 \cdot 12 \pm 6 \cdot 35$ & $5 \cdot 83 \pm 3 \cdot 13$ \\
\hline $\begin{array}{l}\text { Evening }(2200 \mathrm{~h}) \\
\text { salivary cortisol } \\
(\mathrm{nmol} / \mathrm{l})^{\mathrm{a}}\end{array}$ & $4 \cdot 63 \pm 10 \cdot 4$ & $3 \cdot 35 \pm 5 \cdot 61$ \\
\hline
\end{tabular}

All results mean \pm s.D.; $n$, number of participants; $\mathrm{BMI}$, body mass index, WBC, white blood cell count.

${ }^{a}$ The number of participants included in the analyses were 21 males and 15 females. No significant differences were observed between male and female participants. significantly associated with higher concentrations of complement proteins C3 $(P \leq 0 \cdot 001)$ and $\mathrm{C} 4(P \leq 0 \cdot 01)$; increased monocyte phagocytic activity $(P \leq 0 \cdot 05)$; increased NKT percentage expression $(P \leq 0 \cdot 001)$ and absolute count $(P \leq 0 \cdot 001)$; and higher percentage expression of IL6 by activated monocytes $(P \leq 0 \cdot 05)$. In addition, $\mathrm{T}_{3}$ concentration was positively associated with monocyte count, a finding that approached statistical significance $(P=0 \cdot 051)$, and also demonstrated a significant inverse association with the percentage expression of early lymphocyte apoptosis $(P \leq 0 \cdot 05)$.

$\mathrm{T}_{4}$ concentration was closely related to immune markers involved in both innate and adaptive immunity. Higher $\mathrm{T}_{4}$ concentrations $(>85.3 \mathrm{nmol} / \mathrm{l})$ were significantly associated with higher concentrations of complement proteins C3 $(P \leq 0 \cdot 001)$ and $\mathrm{C} 4(P \leq 0 \cdot 05)$; higher CRP concentration $(P \leq 0 \cdot 05)$; and higher neutrophil counts $(P \leq 0 \cdot 001) . \mathrm{T}_{4}$ was also positively correlated with the percentage expression of total memory T-lymphocytes $(P \leq 0 \cdot 01)$; the percentage expression of $\mathrm{CD} 3+/ \mathrm{CD} 4+/ \mathrm{CD} 45 \mathrm{RO}+$ memory T-helper lymphocytes $(P \leq 0.05)$; and the percentage expression of CD3+/CD8 +/CD45RO+memory T-cytotoxic (CTL) lymphocytes $(P \leq 0 \cdot 05)$. In addition, $\mathrm{T}_{4}$ concentration was inversely correlated with the ratio of naive T-cytotoxic to memory T-cytotoxic lymphocytes $(P \leq 0 \cdot 05)$.

A higher $T_{3}: T_{4}$ ratio $(>0 \cdot 23)$ was associated with increased monocyte phagocytic activity $(P \leq 0 \cdot 05)$; higher IL2 receptor density on CD3 + T-lymphocytes $(P \leq 0 \cdot 05)$; and higher absolute counts of NKT cells $(P \leq 0 \cdot 05)$. Moreover, a higher $\mathrm{T}_{3}: \mathrm{T}_{4}$ ratio was also associated with lower neutrophil counts $(P \leq 0 \cdot 05)$.

\section{Discussion}

To our knowledge this is the first study to examine associations between thyroid hormone concentration and markers of immunity in healthy men and women, using a comprehensive panel of immune markers. Thyroid hormone 
Table 3 Associations between serum thyroid hormones concentration and immune function in healthy individuals, aged 55-70 years ${ }^{\mathrm{a}}$

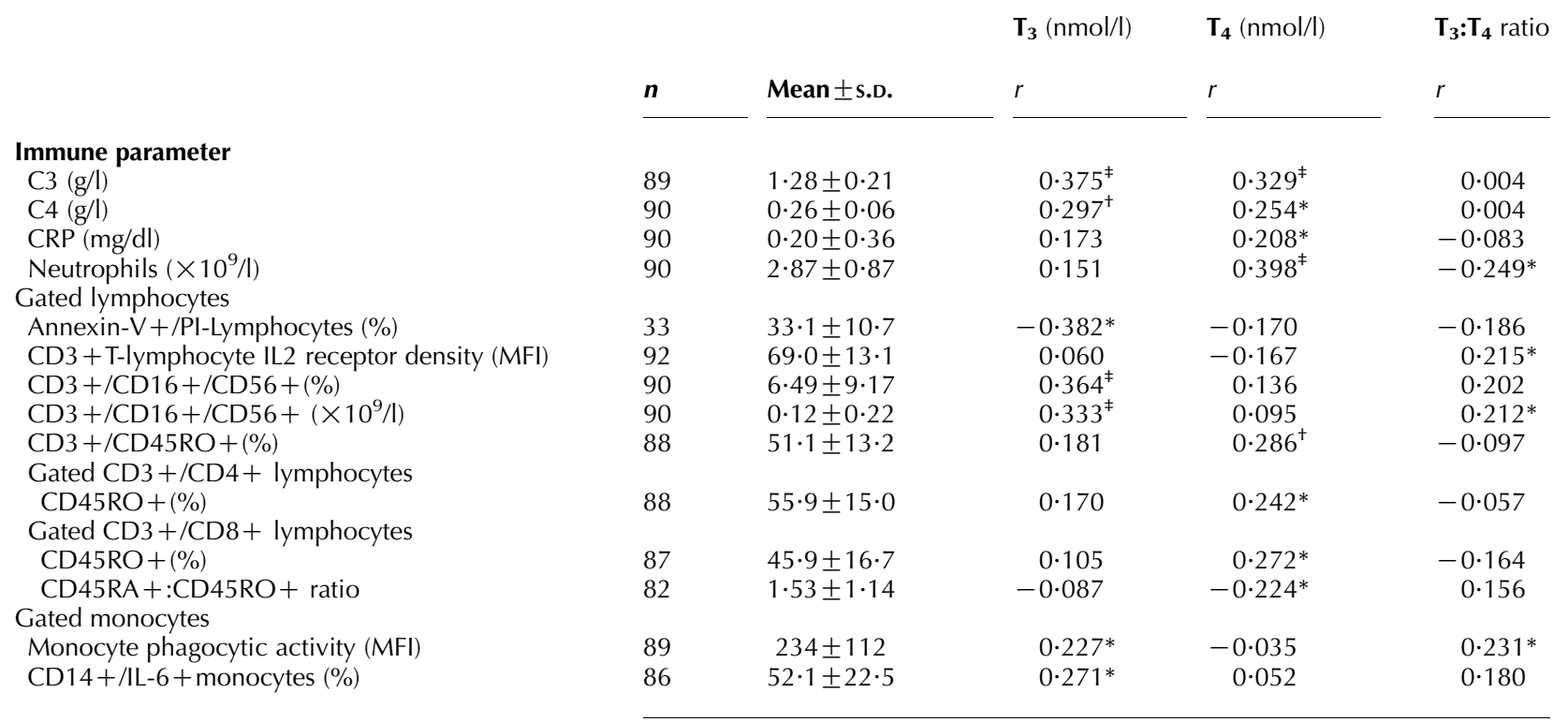

$n$, number of participants; $\mathrm{CD}$, cluster of differentiation. ${ }^{*} P \leq 0 \cdot 05,{ }^{\dagger} P \leq 0 \cdot 01,{ }^{\ddagger} P \leq 0 \cdot 001$.

${ }^{a}$ As age was a significant determinant of immune function, age was used as a co-factor in all correlations between thyroid hormone concentration (total triiodothyronine, $\mathrm{T}_{3}$; total thyroxine, $\mathrm{T}_{4}$ ) and immune markers.

concentration and markers of immunity were within normal physiological ranges. Overall, the current study provides preliminary evidence to suggest that higher concentrations of $\mathrm{T}_{3}$ and $\mathrm{T}_{4}$, within normal physiological ranges, enhance innate and adaptive immunity through maintenance of specific cell populations and greater responsiveness to immune stimuli.

Our observation of a positive relationship between thyroid hormone concentration and markers of inflammation is supported by evidence for the binding of $\mathrm{C} 4$ to thyroperoxidase (TPO), a key enzyme required for thyroid hormone synthesis, that activates the complement cascade (Blanchin et al. 2003). Furthermore, in vivo clinical studies have indicated that altered thyroid hormone concentration is associated with serum IL6 concentration (Bartalena et al. 1994a,b). Furthermore, ex vivo studies have shown that thyroid hormones stimulate free-radical production in polymorphonuclear leukocytes (Mezosi et al. 2005), and that phagocytosis is impaired in hypothyroid patients (Palmblad et al. 1981). Our observations show that NKT cell numbers are associated with higher thyroid hormone concentrations within the normal physiological range. Altered NKT cell numbers and function are associated with increased incidence of autoimmune disorders (Miyake \& Yamamura 2007), and increased NKT cell numbers have been observed in successfully aged individuals with normal thyroid function (Paolisso et al. 2000, Mocchegiani \& Malavolta 2004, Abedin et al. 2005). However, studies in non-autoimmune thyroid disorders are necessary to clarify the relationship between altered thyroid hormone concentration and NKT biology.
Previous studies have reported elevated levels of soluble IL-2R in hyperthyroid patients (Nakanishi et al. 1991), and after oral $\mathrm{T}_{3}$ administration (Mariotti et al. 1992). Our observation of increased surface IL-2R density on lymphocytes in healthy individuals with a higher $\mathrm{T}_{3}: \mathrm{T}_{4}$ ratio suggests that higher concentrations of the metabolically active thyroid hormone $\mathrm{T}_{3}$, within normal physiological concentrations, may contribute to the expression of IL-2R on lymphocytes. $\mathrm{T}_{3}$ was also associated with lower incidence of early lymphocyte apoptosis indicating that this thyroid hormone may also facilitate maintenance of the lymphocyte population. Although evidence for thyroid hormone regulation of lymphocyte responses has been demonstrated previously in animal models (Klecha et al. 2000, 2006), the underlying cellular mechanisms are not clearly understood.

The current study included an extensive panel of phenotypic markers of immunity that provided comprehensive information on immune status and, importantly, functional assays were included which are associated with clinical endpoints and also present a mechanistic understanding of the immune response (Albers et al. 2005). While, clearly as an observational study, the current study cannot discriminate cause-and-effect relationships between immune and endocrine function, it does highlight the requirement for further studies of these complex interactions in healthy individuals. Future studies should include a comprehensive assessment of immune status as described in the current study, but would benefit from the addition of further functional markers such as NK cell activity, phagocyte oxidative burst and the measurement of other pro- and anti-inflammatory 
cytokines. To place the effects of $\mathrm{T}_{3}$ and $\mathrm{T}_{4}$ concentration on immune function in context free $\mathrm{T}_{3}$ and $\mathrm{T}_{4}, \mathrm{TSH}$, antiTPO, antiTG, ACTH and lymphocyte TR assessment should also be incorporated into study designs to assess thyroid and adrenal gland function in relation to HPA- and HPT-axis activity.

In summary, the current study suggests a role for thyroid hormones, within the normal physiological range, in the maintenance of lymphocyte subpopulations, and in mediating the inflammatory response. This study suggests that some of the same immune alterations observed in thyroid disorders are also observed under normal physiological conditions, albeit within normal reference ranges. These preliminary findings indicate the potential benefit of maintaining optimal thyroid hormone concentrations for the maintenance of immune function with advancing age. Overall, the study reveals a complex relationship between the endocrine and immune systems that warrants further study in this vulnerable population of individuals.

\section{Declaration of interest}

The author's declare that there is no conflict of interest that could be perceived as prejudicing the impartiality of the research reported.

\section{Funding}

This work was supported by the European Commission 'Quality of Life and Management of Living Resources' Fifth Framework Program (Contract no: QLK1-CT-2001-00168) and the Department of Employment and Learning (DELNI), UK.

\section{Acknowledgements}

We would like to extend our many thanks to Mary Kelly, University of Ulster, for her role in volunteer recruitment and management; to Prof. Denis Alexander, Belfast City Hospital, Northern Ireland, for his technical advice in flow cytometry; to the Causeway Laboratory, Causeway NHSS Trust, Coleraine, Northern Ireland and to Grenoble University Hospital, France for their assistance with blood analysis; and to all the study participants.

\section{References}

Abedin S, Michel J, Lemster B \& Vallejo A 2005 Diversity of the NKR expression in aging $\mathrm{T}$ cells and in $\mathrm{T}$ cells of the aged: the new frontier into the exploration of protective immunity in the elderly. Experimental Gerontology 40 537-548.

Albers R, Antoine J-M, Bourdet-Sicard R, Calder PC, Gleeson M, Lesourd B, Samartin S, Sanderson IR, Van Loo J, Van Dias FW et al. 2005 Markers to measure immunomodulation in human nutrition intervention studies. British Journal of Nutrition 94 452-481.

Bartalena L, Brogioni S, Grasso L, Rago T, Vitti P, Pinchera A \& Martino E 1994a Interleukin-6: a marker of thyroid-destructive process. Journal of Clinical Endocrinology and Metabolism 79 1424-1427.

Bartalena L, Brogioni S, Grasso L, Velluzzi F \& Martino E $1994 b$ Relationship of the increased serum interleukin- 6 concentrations to changes of thyroid function in nonthyroidal illness. Journal of Endocrinological Investigation 17 269-274
Bellinger D, Thyagarajan S, Lorton D, Maddan K, Tran L \& Felten D 2002 Psychoneuroimmunology today: mechanisms mediating the effects of psychological status on the immune system. In The Psychoimmunology of Cancer, 2 edn, pp 4-62. Eds C Lewis, R O’Brien \& J Barraclough. Oxford: Oxford University Press.

Bisset LR, Lung TL, Kaelin M, Ludwig E \& Dubs RW 2004 Reference values for peripheral blood lymphocyte phenotypes applicable to the healthy adult population in Switzerland. European Journal of Haematology 72 203-212.

Blanchin S, Estienne V, Durand-Gorde J-M, Carayon P \& Ruf J 2003 Complement activation by direct $\mathrm{C} 4$ binding to thyroperoxidase in Hashimoto's thyroiditis. Endocrinology 144 5422-5429.

Bossowski A, Urban M \& Stasiak-Barmuta A 2003 Analysis of changes in the percentage of $\mathrm{B}(\mathrm{CD} 19)$ and $\mathrm{T}$ (CD3) lymphocytes, subsets CD4, CD8 and their memory (CD45RO), and naive (CD45RA) $\mathrm{T}$ cells in children with immune and non-immune thyroid diseases. Journal of Pediatric Endocrinology and Metabolism 16 63-70.

Chakraborti S, Chakraborti T, Mandal M, Das S \& Batabyal SK 1999 Hypothalamic-pituitary-thyroid axis status of humans during development of ageing process. Clinica Chimica Acta 288 137-145.

Chiovato L, Mariotti S \& Pinchera A 1997 Thyroid diseases in the elderly. Bailliere's Clinical Endocrinology and Metabolism 11 251-270.

Covas MI, Esquerda A, Garcia-Rico A \& Mahy N 1992 Peripheral blood T-lymphocyte subsets in autoimmune thyroid disease. Journal of Investigational Allergology \& Clinical Immunology 2 131-135.

Csaba G, Kovacs P \& Pallinger E 2005 Effect of the inhibition of triiodothyronine $\left(T_{3}\right)$ production by thiamazole on the $T_{3}$ and serotonin content of immune cells. Life Sciences 76 2043-2052.

Davis SL 1998 Environmental modulation of the immune system via the endocrine system. Domestic Animal Endocrinology 15 283-289.

Dorshkind K \& Horseman ND 2000 The roles of prolactin, growth hormone, insulin-like growth factor-I, and thyroid hormones in lymphocyte development and function: insights from genetic models of hormone and hormone receptor deficiency. Endocrine Reviews 21 292-312.

Dorshkind K \& Horseman N 2001 Anterior pituitary hormones, stress, and immune system homeostasis. BioEssays 23 288-294.

Fabris N, Mocchegiani E \& Provinciali M 1995 Pituitary-thyroid axis and immune system: a reciprocal neuroendocrine-immune interaction. Hormone Research 43 29-38.

Felten DL \& Felten SY 1991 The innervation of lymphoid tissue. In Psychoneuroimmunology, 2 edn, pp 27-69. Eds R Ader, DL Felten \& N Cohen. New York: Academic press.

Hodkinson CF, O'Connor JM, Alexander HD, Bradbury I, Bonham MP, Hannigan BM, Gilmore WS, Strain JJ \& Wallace JMW 2006 Whole blood analysis of phagocytosis, apoptosis, cytokine production and leukocyte subsets in healthy older men and women: the ZENITH study. Journals of Gerontology. Series A, Biological Sciences and Medical Sciences 61 907-917.

Klecha AJ, Genaro AM, Lysionek AE, Caro RA, Coluccia AG \& Cremaschi GA 2000 Experimental evidence pointing to the bidirectional interaction between the immune system and the thyroid axis. International Journal of Immunopharmacology 22 491-500.

Klecha AJ, Genaro AM, Gorelik G, Barreiro Arcos ML, Silberman DM, Schuman M, Garcia SI, Pirola C \& Cremaschi GA 2006 Integrative study of hypothalamus-pituitary-thyroid-immune system interaction: thyroid hormone-mediated modulation of lymphocyte activity through the protein kinase C signaling pathway. Journal of Endocrinology 189 45-55.

Klein JR 2006 The immune system as a regulator of thyroid hormone activity. Experimental Biology and Medicine 231 229-236.

Kretowski A, Mysliwiec J, Turoswki D, Wysocka J \& Kinalska I 1999 Analysis of recently activated, memory and naive lymphocyte $\mathrm{T}$ subsets in the peripheral blood of patients with Graves' disease and insulin-dependent diabetes mellitus. Roczniki Akademii Medycznej w Bialymstoku 44 226-234.

Latrofa F \& Pinchera A 2005 Aging and the thyroid. Hot Thyroidology 1 http://www.hotthyroidology.com/print.php?ID =148

Ligthart GJ, Corberand JX, Fournier C, Galanaud P, Hijmans W, Kennes B, Müller-Hermelink HK \& Steinmann GG 1984 Admission criteria for immunogerontological studies in man: the senieur protocol. Mechanisms of Ageing and Development 28 47-55. 
Magri F, Muzzoni B, Cravello L, Fioravanti M, Busconi L, Camozzi D, Vignati G \& Ferrari E 2002 Thyroid function in physiological aging and in centenarians: possible relationship with some nutritional markers. Metabolism: Clinical and Experimental 51 105-109.

Mariotti S, Caturegli P, Barbesino G, Marino M, Del Prete GF, Chiovato L, Tonacchera M, DeCarli M \& Pinchera A 1992 Thyroid function and thyroid autoimmunity independently modulate serum concentration of soluble interleukin 2 (IL-2) receptor (sIL-2R) in thyroid diseases. Clinical Endocrinology 37 415-422.

Mariotti S, Franceschi C, Cossarizza A \& Pinchera A 1995 The aging thyroid. Endocrine Reviews 16 686-715.

McNerlan SE, Alexander HD \& Rea IM 1999 Age-related reference intervals for lymphocyte subsets in whole blood of healthy individuals. Scandinavian Journal of Clinical and Laboratory Investigation 59 89-92.

Mezosi E, Szabo J, Nagy EV, Borbely A, Varga E \& Paragh G 2005 Nongenomic effect of thyroid hormone on free-radical production in human polymorphonuclear leukocytes. Journal of Endocrinology 185 121-129.

Miyake S \& Yamamura T 2007 NKT cells and autoimmune diseases: unraveling the complexity. Current Topics in Microbiology and Immunology 314 251-267.

Mocchegiani E \& Malavolta M 2004 NK and NKT cell functions in immunosenescence. Aging Cell 3 117-184.

Mooradian AD 1995 Normal age-related changes in thyroid hormone economy. Clinics in Geriatric Medicine 11 159-169.

Nakanishi K, Taniguchi Y \& Ohta Y 1991 Increased soluble interleukin 2 receptor levels in autoimmune thyroid disease. Acta Endocrinologica 125 253-258.

Pallinger E, Kovacs P \& Csaba G 2005 Presence of hormones (triiodothyronine, serotonin and histamine) in the immune cells of newborn rats. Cell Biology International 29 826-830.
Palmblad J, Adamson U, Roseenqvist U, Uden AM \& Venizelos N 1981 Neutrophil function in hypothyroid patients. Acta Medica Scandinavica 210 287-291.

Paolisso G, Barbieri M, Bonafe M \& Franceschi C 2000 Metabolic age modelling: the lesson from centenarians. European Journal of Clinical Investigation 30 888-894.

Ribeiro-Carvalho MM, Smaniotto S, Neves-dos-Santos S, Mouço T, Savino W \& Mello-Coelho V 2007 Triiodothyronine modulates differential homing of recent thymic emigrants to peripheral lymphoid organs. Scandinavian Journal of Immunology 66 8-16.

Santagostino A, Garbaccio G, Pistorio A, Bolis V, Camisasca G, Pagliaro P \& Girotto M 1999 An Italian national multicenter study for the definition of reference ranges for normal values of peripheral blood lymphocyte subsets in healthy adults. Haematologica 84 499-504.

Stulnig T, Maczek C, Bock G, Majdic O \& Wick G 1995 Reference intervals for human peripheral blood lymphocyte subpopulations from 'healthy' young and aged subjects. International Archives of Allergy and Immunology 108 205-210.

Yen P 2001 Physiological and molecular basis of thyroid hormone action. Physiological Reviews 81 1097-1142.

Received in final form 12 April 2009
Accepted 27 April 2009

Made available online as an Accepted Preprint 27 April 2009 